\title{
Electrochemical and Mechanical Failure of Graphite-Based Anode Materials in Li-Ion Batteries for Electric Vehicles
}

\author{
Cheng Lin, ${ }^{1}$ Aihua Tang, ${ }^{1,2}$ Ningning $\mathrm{Wu}^{3}{ }^{3}$ and Jilei Xing ${ }^{1}$ \\ ${ }^{1}$ National Engineering Laboratory for Electric Vehicles and Collaborative Innovation Center of Electric Vehicles in Beijing, \\ School of Mechanical Engineering, Beijing Institute of Technology, Beijing 100081, China \\ ${ }^{2}$ Sichuan Provincial Key Lab of Process Equipment and Control, School of Mechanical Engineering, \\ Sichuan University of Science \& Engineering, Zigong 643000, China \\ ${ }^{3}$ Citic Guoan Mengguli Power Source Technology Co., Ltd, Beijing 102200, China
}

Correspondence should be addressed to Aihua Tang; tahme@163.com

Received 5 June 2016; Revised 16 August 2016; Accepted 18 September 2016

Academic Editor: David Sebastián

Copyright (C) 2016 Cheng Lin et al. This is an open access article distributed under the Creative Commons Attribution License, which permits unrestricted use, distribution, and reproduction in any medium, provided the original work is properly cited.

\begin{abstract}
Graphite-based anode materials undergo electrochemical reactions, coupling with mechanical degradation during battery operation, can affect or deteriorate the performance of Li-ion batteries dramatically, and even lead to the battery failure in electric vehicle. First, a single particle model (SPM) based on kinetics of electrochemical reactions was built in this paper. Then the Liion concentration and evolution of diffusion induced stresses (DISs) within the SPM under galvanostatic operating conditions were analyzed by utilizing a mathematical method. Next, evolution of stresses or strains in the SPM, together with mechanical degradation of anode materials, was elaborated in detail. Finally, in order to verify the hypothesis aforementioned surface and morphology of the graphite-based anode dismantled from fresh and degraded cells after galvanostatic charge/discharge cycling were analyzed by X-ray diffraction (XRD), field-emission scanning electron microscopy (SEM), and transmission electron microscopy (TEM). The results show that large volume changes of anode materials caused DISs during Li-ion insertion and extraction within the active particles. The continuous accumulations of DISs brought about mechanical failure of the anode eventually.
\end{abstract}

\section{Introduction}

Li-ion cells are very compelling candidates for power supplies with their high-power and energy density and low selfdischarge rate. They have been widely used in new-energy vehicles such as battery electric vehicles (BEV), hybrid electrical vehicles (HEV), and plug-in hybrid electric vehicles (PHEV) in the past few decades [1]. For the health management study of Li-ion batteries in electrical vehicles, knowledge about the mechanical degradation of anode materials under certain operating conditions is of great significance [2]. Mechanical degradation may appear owing to deformations and stress generation as lithium ions intercalate/deintercalate into/from the active anode and cathode materials during charging and discharging operations over time. It is important to understand and mitigate mechanical electrode degradation in Li-ion batteries for EVs since it can accelerate battery aging and further lead to failure [3].
Unfortunately, the mechanical degradation under some special working conditions is one of the critical challenges in improving performance and prolonging life span of the cells at present. The difficulties existing in measurement of the critical properties and phenomena in electrode materials instigated extensive research activities on numerical modeling of $\mathrm{Li}$-ion batteries worldwide in the last few years [35]. Christensen and Newman [6] developed a mathematical model based upon concentrated solution theory and porous electrode theory that calculate the volume changes and simulate the distribution profiles of Li-ion concentration and stress during the repeated lithium insertion/extraction processes into/from a spherical electrode particle. Doyle et al. [7] presented numerical models with a finite-volume method and porous electrode theory which predict the transport, kinetics, and reaction processes in batteries. These models or methods have shown good agreement with discharge experiments to a certain extent; however, they cannot ideally 
account for mechanical failure of active materials within the electrodes in batteries applied in EVs. Cheng and Verbrugge [4] recently analyzed the evolution of stress and strain energy due to DIS in a spherical insertion electrode particle under either galvanostatic or potentiostatic condition. Deshpande and coworkers $[8,9]$ established relationships between surface stress, surface energy, and the magnitude of DISs in nanowires and investigated the effects of surface elasticity and surface energy on the stress evolution in spherical electrodes. They also correlated elastic strain energy, DISs, and stress discontinuities at the phase boundaries of the different phases that form during lithiation/delithiation using a core-shell structural model [10].

The literature on modeling of stress and crack formation within active electrode particles is quite extensive. Stresses and fracture in electrodes undergoing volume changes were predicted in a one-dimensional model by Huggins and Nix in [11]. Furthermore, a terminal particle size below which particles are not expected to crack was also predicted in their model. Crack formation of electrode particles was modeled with facture mechanics by Aifantis and Dempsey [12]. Other researchers [4, 13-21] noted that the maximum stress within active particles is in proportion to the product of particle size and the concentration gradient. The maximum stress increases with current density, radius of spherical particles, and lower Li-diffusivity in the electrode material, all of which lead to steeper concentration gradients between the surface and the core. They were also verified in literatures [6, 9-11].

Despite significant advances in the theoretical investigation of stress and strain evolution within electrode active materials of Li-ion batteries, some innovative researches are still needed to explore the mechanisms of mechanical failure. In this paper, the SPM was established in Section 2. The solutions of the diffusion equations with initial and boundary conditions and analytic expression of evolution of DISs for the galvanostatic control within the SPM were presented in Section 3. In Section 4, a commercial Li-ion battery was cycled in a constant current (CC) charge/discharge process with a battery cycler in the potential range of $3.0 \mathrm{~V}-4.2 \mathrm{~V}$. For comparison, the other fresh battery belonging to the same batch was disassembled in this paper. The anodes dismantled from fresh and degraded batteries were analyzed by XRD, SEM, and TEM. The measurements from XRD reveal the lattice parameter changes, and SEM and TEM were applied to verify the microstructure differences of the graphitic nanoparticles between the fresh and degraded anodes. These are the main contributions and are helpful to understand the DISs in this manuscript. Besides, the complex interplay between the origins and evolution of mechanical degradation and structure changes during electrochemical reaction in electrode materials were also discussed.

\section{Single Particle Electrode Model}

The single particle model (SPM) was first proposed by Haran et al. [22] in determination of the hydrogen diffusion coefficient within a metal hydride electrode and used to calculate the Li-ion diffusion of the insertion electrode during discharging/charging process [23]. A schematic illustration of electrochemical-based Li-ion battery is depicted in Figure 1. The Li-ion battery is considered as three parts: negative electrode (graphite anode), separator, and positive electrode (metallic oxide cathode), as shown in Figure 1 with single spherical particle representing respective electrode of Li-ion batteries in the discharge/charge process according to [2426]. During charge process, Li-ions diffuse to the surface of metallic oxide particles in the cathode, where they extract the lithiated particles, travel through the electrolyte separator, and are inserted and diffuse in solid phase of graphite particles in the anode. Simultaneously, electrons emitted from the cathode transmit through the external circuit towards the anode. During discharge, positive Li-ions and electrons flow in the reverse direction; see Figure 1.

\section{Evolution of Stresses within a SPM}

For a spherical particle, several groups provided evidence for lithium ion diffusion and phase transformations in mechanical properties. Qi et al. [27] showed that material properties change substantially upon lithium insertion. Deshpande et al. $[9-11,15]$ considered the effects of elastic properties dependent on solute concentration on diffusion induced stresses in single phase systems. Furthermore, analytic solutions for stresses [3, 4, 8-11, 14-17, 19-23] can help elucidate the effects of lithium ions diffusion on DISs. We study DISs within a SPM caused by lithium insertion and extraction in Li-ion battery electrodes under galvanostatic charging strategies.

3.1. Solid Mechanics of DIS. The stresses induced by Li-ion diffusion are considered in a SPM of radius $R$. The analogy between thermal and DIS is based on the assumption that the bulk of the spherical SP is an isotropic linear elastic solid. The expressions of stress-strain relationships are established for the radial and tangential components in the spherical coordinate system by employing the analogy [4, 8-11, 20, 21]:

$$
\begin{aligned}
& \varepsilon_{r}=\frac{1}{E}\left(\sigma_{r}-2 \nu \sigma_{\theta}\right)+\frac{1}{3} \Omega c, \\
& \varepsilon_{\theta}=\frac{1}{E}\left[(1-\nu) \sigma_{\theta}-\nu \sigma_{r}\right]+\frac{1}{3} \Omega c,
\end{aligned}
$$

where $E$ is Young's modulus, $\nu$ is Poisson's ratio, $\Omega$ is the partial molar volume of the solute, and $c$ is the molar concentration. Besides, the elastic properties are assumed to be constant and have nothing to do with the concentration $c$.

For infinitely small formulation of deformation, the radial and tangential strains of the spherically symmetric particle are given by the following:

$$
\begin{aligned}
& \varepsilon_{r}=\frac{d \mu}{d r}, \\
& \varepsilon_{\theta}=\frac{\mu}{r},
\end{aligned}
$$

where $\mu$ is the radial displacement. As atomic diffusion is much slower than rates of elastic deformation in solids, transients are often neglected in solving the problem of solid 


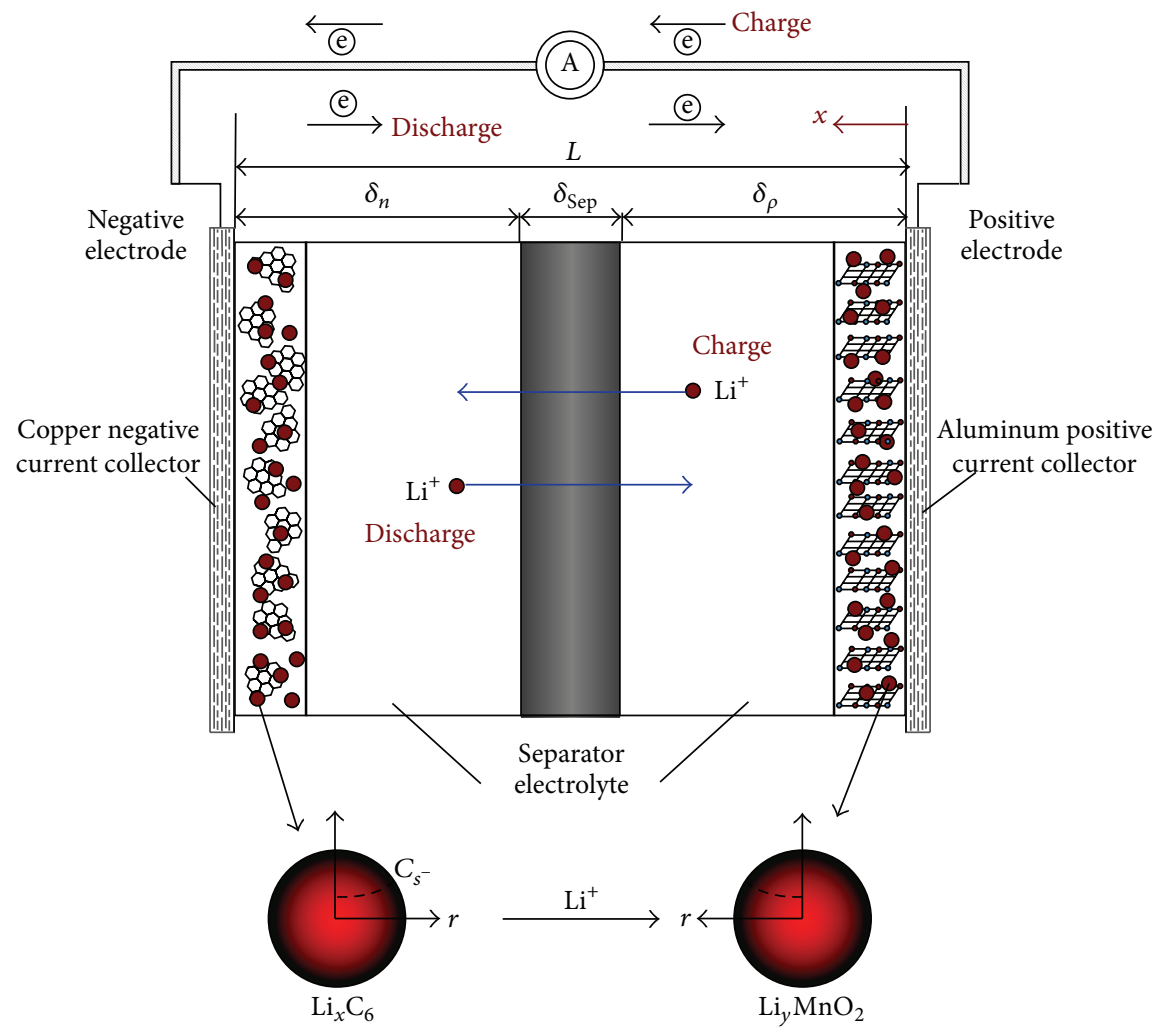

FIGURE 1: Schematic illustration of a Li-ion battery during discharge/charge.

mechanics. Without any body-force, the static mechanical equilibrium equation within a sphere is listed by [28]

$$
\frac{d \sigma_{r}}{d r}+\frac{2}{r}\left(\sigma_{r}-\sigma_{\theta}\right)=0
$$

The boundary condition $\sigma_{r}(R)=0$ and remains finite at $r=0$, and the solutions for radial and tangential stresses are listed by

$$
\begin{aligned}
\sigma_{r}(r) & =\frac{2}{9} E \Omega(1-\nu)^{-1}\left[c_{\mathrm{av}}(R)-c_{\mathrm{av}}(r)\right], \\
\sigma_{\theta}(r) & =\frac{E \Omega}{9}(1-\nu)^{-1}\left[2 c_{\mathrm{av}}(R)+c_{\mathrm{av}}(r)-3 c(r)\right],
\end{aligned}
$$

where $c_{\mathrm{av}}(r) \equiv\left(3 / r^{3}\right) \int_{0}^{r} r^{2} c(r) d r$ is the average solute concentration of the spherical volume within the particle of radius $R$.

One principal shear stress equals zero and the other two are both $\left(\sigma_{r}-\sigma_{\theta}\right) / 2$ for the spherical symmetry, and the principal shear stress is given by

$$
\frac{\sigma_{r}(r)-\sigma_{\theta}(r)}{2}=\frac{E \Omega}{6}(1-v)\left(c(r)-c_{\mathrm{av}}(r)\right) .
$$

Thus, the stresses distributed at any given time and location can be obtained under the conditions that the concentration distribution is known [4].
3.2. DIS under Galvanostatic Operation Conditions. The stress evolution within the spherical particles under galvanostatic condition is studied in this paper since Li-ion batteries applied in EVs are mostly charged with a typical constant current constant voltage (CC-CV) charging strategy. A simple relevant equation of Li-ion diffusion within a spherical SP of radius $R$ is listed in the spherical coordinate system by $[29,30]$

$$
\frac{\partial c}{\partial t}=\frac{D}{r^{2}} \frac{\partial}{\partial r}\left(r^{2} \frac{\partial c}{\partial r}\right)
$$

where symbol $D$ represents constant diffusion coefficient.

Considering that the particle parameters of different batteries will be different, (6) would be transformed into dimensionless form as follows:

$$
\begin{aligned}
X & =\frac{r}{R}, \\
\tau & =\frac{D t}{R^{2}}, \\
y & =\frac{c-c_{0}}{c_{R}-c_{0}} .
\end{aligned}
$$

Then, the governing equation (6) in dimensionless form is shown as follows:

$$
\frac{\partial y}{\partial \tau}=\frac{1}{X^{2}} \frac{\partial}{\partial X}\left(X^{2} \frac{\partial y}{\partial X}\right) .
$$

This condition denotes that the current is a constant and the ionic flux is invariant at electrode surface. The initial and 
boundary conditions subjected to galvanostatic control in dimensionless form are given by

$$
\begin{gathered}
y(0, X)=y_{0}, \quad \tau=0, \quad 0 \leq X \leq 1, \\
\left.\frac{\partial y(\tau, X)}{\partial X}\right|_{X=0}, \quad \tau \geq 0, \\
\left.\frac{\partial y(\tau, X)}{\partial X}\right|_{X=1}=\frac{I}{F}, \quad \tau \geq 0 .
\end{gathered}
$$

The analytic solution to address the diffusion equation (8) with initial and boundary conditions (see (10)) subjected to galvanostatic control is as follows $[29,30]$ :

$$
\begin{aligned}
y & =\frac{I R}{\operatorname{FD}\left(c_{R}-c_{0}\right)}\left(3 \tau+\frac{1}{2} X^{2}-\frac{3}{10}\right. \\
& \left.-\frac{2}{X} \sum_{n=1}^{\infty}\left(\lambda_{n}^{-2} \csc \left(\lambda_{n}\right) \sin \left(\lambda_{n} X\right) \exp \left(-\lambda_{n}^{2} \tau\right)\right)\right),
\end{aligned}
$$

where $\lambda_{n}(n=1,2,3, \ldots)$ are the positive roots of $\tan \left(\lambda_{n}\right)=$ $n$.

By utilizing (10), the average concentrations of Li-ions can be expressed as

$$
\begin{aligned}
& y_{\mathrm{av}}(\tau, X)=\frac{3 I R}{F D\left(c_{R}-c_{0}\right)}\left[\tau+\frac{1}{10}\left(X^{2}-1\right)-\frac{2}{X^{3}}\right. \\
& \cdot \sum_{n=1}^{\infty}(n \pi)^{-4} \csc (n \pi) \\
& \left.\cdot\left(\sin (n \pi X)-(n \pi X) \cos (n \pi X) \exp \left(-n^{2} \pi^{2} \tau\right)\right)\right], \\
& y_{\mathrm{av}}(\tau, 1)=\frac{3 I R \tau}{F D\left(c_{R}-c_{0}\right)} .
\end{aligned}
$$

Equation (11) can be used to solve for stresses after (3) is substituted into it:

$$
\begin{aligned}
& \frac{3 \sigma_{r}(1-\nu) F D}{E \Omega I R}=\frac{1}{5}\left(1-X^{2}\right)+\frac{4}{X^{3}} \sum_{n=1}^{\infty}(n \pi)^{-4} \csc (n \pi) \\
& \cdot\left(\sin (n \pi X)-(n \pi X) \cos (n \pi X) \exp \left(-n^{2} \pi^{2} \tau\right)\right) \\
& =\xi_{r}^{I}(\tau, X), \\
& \frac{3 \sigma_{\theta}(1-\nu) F D}{E \Omega I R}=\frac{1}{5}\left(1-2 X^{2}\right) \\
& +2 \sum_{n=1}^{\infty} \frac{\exp \left(-n^{2} \pi^{2} \tau\right)}{n \pi \sin (n \pi)}(n \pi X)^{-1}(\sin (n \pi X) \\
& \left.-(n \pi X)^{-2} \sin (n \pi X)-(n \pi X)^{-1} \cos (n \pi X)\right) \\
& =\xi_{\theta}^{I}(\tau, X),
\end{aligned}
$$

where $\xi_{r}^{I}(\tau, X)$ and $\xi_{\theta}^{I}(\tau, X)$ are radial and tangential stresses, respectively, in dimensionless form under galvanostatic control. The shear stress is determined by the following:

$$
\begin{gathered}
\frac{3\left(\sigma_{r}-\sigma_{\theta}\right)(1-\nu) F D}{2 E \Omega I R}=\frac{X^{2}}{10}+\frac{3}{X^{3}} \sum_{n=1}^{\infty}(n \pi)^{-4} \csc (n \pi) \\
\cdot\left(\sin (n \pi X)-(n \pi X) \cos (n \pi X) \exp \left(-n^{2} \pi^{2} \tau\right)\right) \\
-\frac{1}{X} \sum_{n=1}^{\infty}(n \pi)^{-2} \csc (n \pi) \sin (n \pi X) \exp \left(-n^{2} \pi^{2} \tau\right) .
\end{gathered}
$$

Figure 2 shows the Li-ion concentration and radial, tangential, and shear stresses of SPM in the insertion process under galvanostatic condition. The algebraic expression, normalized by $(1 / 3) E \Omega I R(F D)^{-1}(1-v)^{-1}$, is a function of position and time.

The current sign in (9) is defined as follows: positive current indicates insertion process and negative current indicates extraction of $\mathrm{Li}$-ions. The $\mathrm{Li}^{+}$concentration increases with position and time as depicted in Figure 2(a). During Li-ion insertion process, the radial stress appears in the form of compressive stress at the center of the sphere and approximates to zero close to the sphere surface. It increases with time near the sphere center and reaches the maximum at the center (see Figure 2(b)).

The tangential stress appears as tensile stress near the sphere center and compressive close to the surface. The maximum of tensile tangential stress appears at the center before the Li-ions reach there and decreases monotonically towards the surface; then, it reverses and appears as compression stress. Afterwards, it increases along the direction of sphere surface. Moreover, the tangential stress increases in magnitude with time at any location and finally tends to a steady-state (see Figure 2(c)). The shear stress reaches the maximum at the location of sphere center, decreases towards the surface for all times, and reaches a steady-state afterwards. In addition, the shear stress decreases before dimensionless time $\tau=D t / R^{2}=0.1$, while it increases after dimensionless time $\tau=D t / R^{2}=0.2$ for all positions within the sphere, as shown in Figure 2(d). Similar conclusions (i.e., compression instead of tension) can be drawn for Li-ion extraction process.

\section{Validation}

To demonstrate the complex causality between electrochemistry and mechanical degradation of anode in lithium ion batteries, we opted to study mesophase-carbon-microbead $(\mathrm{MCMB})$ anode as a representative material that undergoes interactions and subsequent DISs during electrochemical cycling. The investigated commercial batteries used in this work have a normal capacity rating of $87 \mathrm{Ah}$ and contain carbon material in anodes. The fresh battery was first charged at $1 \mathrm{C}$ rate to $4.2 \mathrm{~V}$ and discharged to $3.0 \mathrm{~V}$ subsequently. $1 \mathrm{C}$ rate means the current is $87 \mathrm{~A}$. During each cycle, the battery was operated in a constant current (CC) charge mode and then underwent a CC discharge until its voltage reaches $3.0 \mathrm{~V}$. The rest time between charge and discharge is two hours. The 


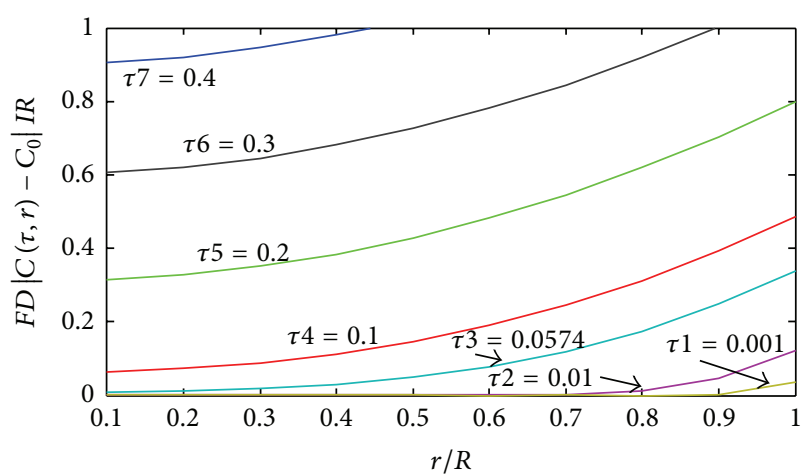

(a) Li-ion concentration

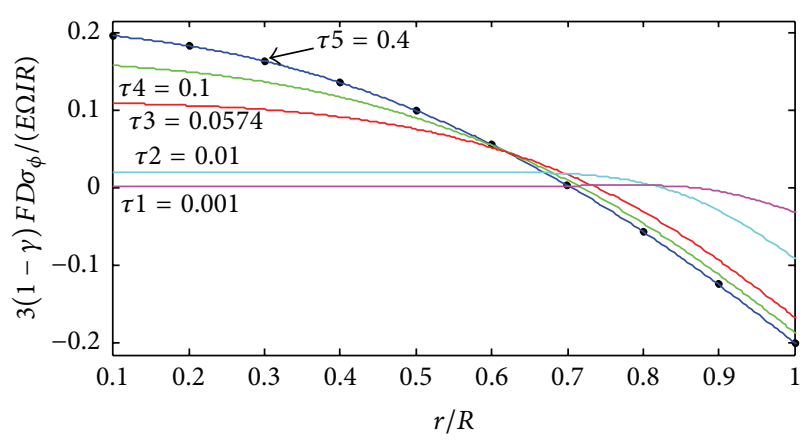

(c) Tangential stress

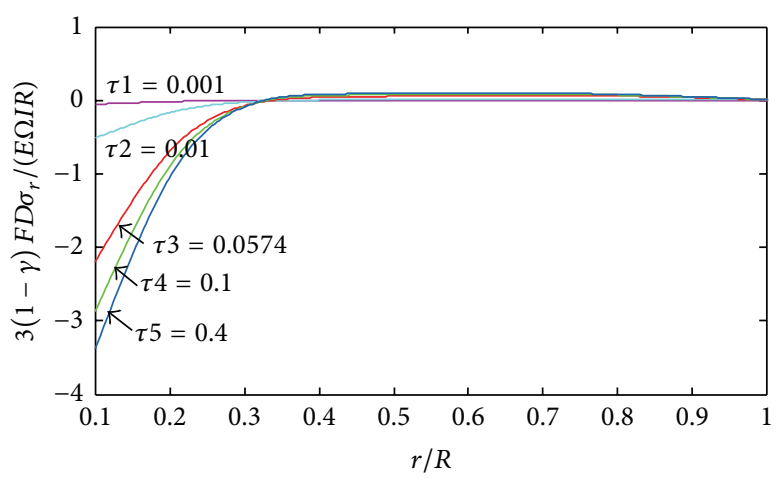

(b) Radial stress

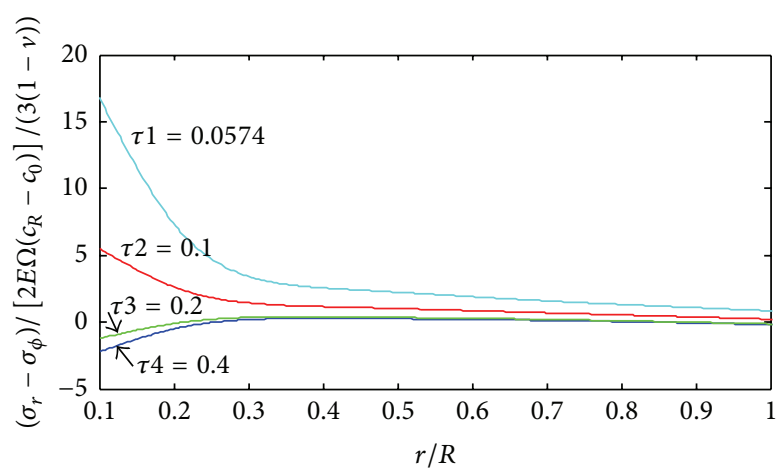

(d) Shear stress

FIGURE 2: Characteristic profile of SPM in the insertion process under galvanostatic control.

stop criterion for the cycling tests is $\mathrm{SOH}=80 \%$ at $25^{\circ} \mathrm{C}$. The surface and structure morphology of anode materials dismantled from fresh and cycled cells were investigated by XRD, SEM, and TEM.

The graphitic MCMB is spherical and contains somewhat randomly oriented graphitic domains. It consists of graphene sheets staggered in either an $\mathrm{AB}$ (hexagonal, the most common form) or $\mathrm{ABC}$ (rhombohedral) stacking arrangement. Upon insertion of lithium ions during charge, the graphite was lithiated, and every fourth layer was filled before the next layers begin to take up lithium. However, lithiation may be initiated at several different sites on the surface of a graphite grain, and the lithiated layers did not need to correspond to one another at different nucleation sites. Hence, the expansion of each grain may represent a large fraction of the expansion of a fully lithiated grain [19]. During discharge, the graphite was delithiated and each grain may embody the contraction of a delithiated grain. The anode film mechanically failed due to the stress induced by the lattice parameter change during Li-ion intercalation/deintercalation. The average grain sizes of nanoparticles on the anode from fresh and degraded cell are compared by $\mathrm{XRD}$ in Figure 3. Thus, the volume changes of the particle during Li-ion insertion and extraction resulted in a strain differential. It accumulated continuously and gave rise to stresses within the particle as the cycle increases [9]. Subsequently, cracks initiated, propagated preferentially at grain boundaries of the particle, and resulted in mechanical

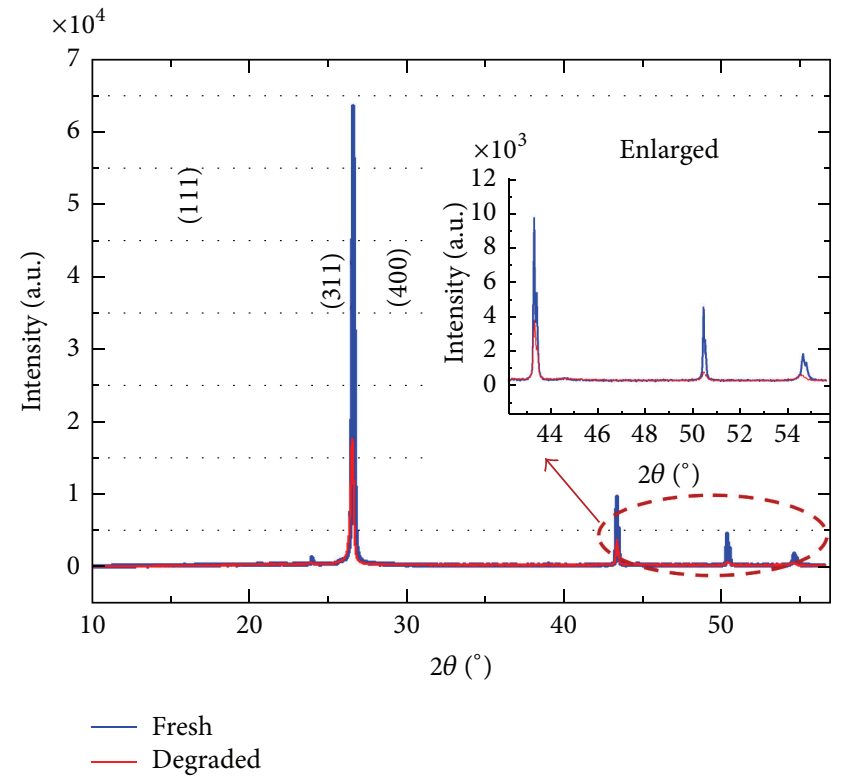

FIGURE 3: XRD spectra of graphite-based anode from fresh and degraded cell.

failure of the anode materials. They were also verified by the SEM and the high-resolution TEM micrographs (Figure 4), with an average particle size of $100 \mathrm{~nm}$, which is in good 


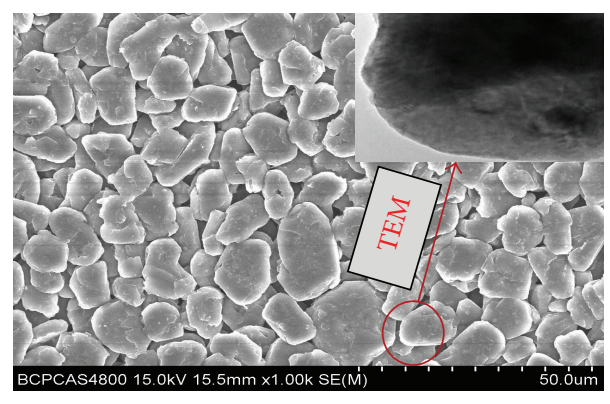

(a) Fresh graphite-based anode

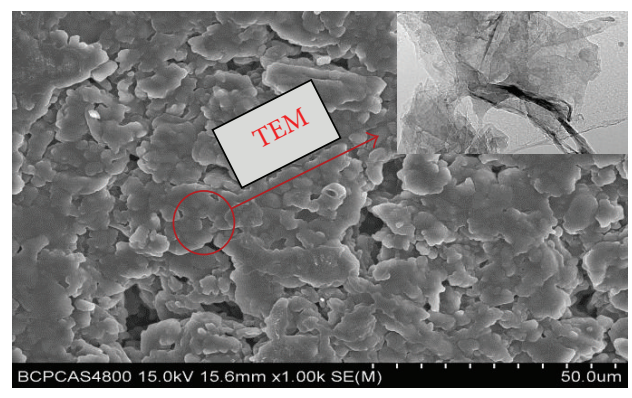

(b) Degraded graphite-based anode

FIGURE 4: SEM images of (a) fresh graphite-based anode before cycling tests and (b) degraded graphite-based anode after 500 cycles.

agreement with the results obtained by XRD [13]. TEM images of lithiated anode display smooth edges of graphite (Figure 4(b)) with a very thin solid electrolyte interphase (SEI) film. The crack and fracture could be observed on cycled anode from the TEM images (Figure 4(b)). The red arrows in Figure 4(b) point to the cracked nanoparticles visible on the graphitic layer surface. Besides, the edge of graphite sheet structure began to mellow and some circular particles with diameter of 100 150 nm appeared on surface of the sheet, which can be associated with the lithium plating and side reaction products produced at the reactions between anode surface and the electrolyte. They also can be observed in SEM and TEM images in Figure 4(b). By a unique combination analysis of SEM, TEM, and XRD, the results suggest that the mechanical failure of anode material can be caused by accumulated DISs within active particles. The stresses are initiated by repeated volume changes while the Li-ions insert and extract the host particles.

\section{Conclusions}

This paper studied the evolution of stresses in a graphitebased anode of Li-ion batteries for EVs considering solid mechanics, diffusion theory, and electrochemical interfacial kinetics under galvanostatic condition. The profiles of $\mathrm{Li}$-ion concentration and radial, tangential, and shear stresses in the SPM were analyzed and presented in the insertion and deinsertion process of the lithium ions in the particles. Furthermore, the evolution of phase structure and morphology for materials with anode demonstrates that a combination of XRD, SEM, and TEM can track the cause and effect of electrochemical and mechanical failure processes in a $\mathrm{Li}$ ion battery for EVs. The experimental and analytical results show that large volume changes of anode materials occur during Li-ion insertion and extraction within the active particles. The accumulated changes lead to stresses within the active particles as the battery cycles, and they further lead to mechanical failure of the anode.

\section{Competing Interests}

The authors declare no competing interests regarding the publication of this article.

\section{Acknowledgments}

This work was supported by the National Natural Science Foundation of China (51575044) and Sichuan Provincial Key Lab of Process Equipment and Control Foundation (GK201603).

\section{References}

[1] H. Rahimi-Eichi, F. Baronti, and M.-Y. Chow, "Online adaptive parameter identification and state-of-charge coestimation for lithium-polymer battery cells," IEEE Transactions on Industrial Electronics, vol. 61, no. 4, pp. 2053-2061, 2014.

[2] L. Zhang, L. Wang, C. Lyu, J. Li, and J. Zheng, "Non-destructive analysis of degradation mechanisms in cycle-aged graphite/ $\mathrm{LiCoO}_{2}$ batteries," Energies, vol. 7, no. 10, pp. 6282-6305, 2014.

[3] R. N. Kuzmin, D. S. Maximov, N. P. Savenkova, and A. V. Shobukhov, "Mathematical modeling of hysteresis in porous electrodes," Journal of Mathematical Chemistry, vol. 50, no. 9, pp. 2471-2477, 2012.

[4] Y.-T. Cheng and M. W. Verbrugge, "Evolution of stress within a spherical insertion electrode particle under potentiostatic and galvanostatic operation," Journal of Power Sources, vol. 190, no. 2, pp. 453-460, 2009.

[5] B. Stiaszny, J. C. Ziegler, E. E. Krauß, M. Zhang, J. P. Schmidt, and E. Ivers-Tiffée, "Electrochemical characterization and postmortem analysis of aged $\mathrm{LiMn}_{2} \mathrm{O}_{4}-\mathrm{NMC} /$ graphite lithium ion batteries part II: calendar aging," Journal of Power Sources, vol. 258, pp. 61-75, 2014.

[6] J. Christensen and J. Newman, "Stress generation and fracture in lithium insertion materials," Journal of Solid State Electrochemistry, vol. 10, no. 5, pp. 293-319, 2006.

[7] M. Doyle, T. F. Fuller, and J. Newman, "Modeling of galvanostatic charge and discharge of the lithium/polymer/insertion cell," Journal of the Electrochemical Society, vol. 140, no. 6, pp. 1526-1533, 1993.

[8] R. Deshpande, Y.-T. Cheng, and M. W. Verbrugge, "Modeling diffusion-induced stress in nanowire electrode structures," Journal of Power Sources, vol. 195, no. 15, pp. 5081-5088, 2010.

[9] R. Deshpande, Y. Qi, and Y.-T. Cheng, "Effects of concentrationdependent elastic modulus on diffusion-induced stresses for battery applications," Journal of the Electrochemical Society, vol. 157, no. 8, pp. A967-A971, 2010. 
[10] R. Deshpande, Y.-T. Cheng, M. W. Verbrugge, and A. Timmons, "Diffusion induced stresses and strain energy in a phasetransforming spherical electrode particle," Journal of the Electrochemical Society, vol. 158, no. 6, pp. A718-A724, 2011.

[11] R. A. Huggins and W. D. Nix, "Decrepitation model for capacity loss during cycling of alloys in rechargeable electrochemical systems," Ionics, vol. 6, no. 1-2, pp. 57-63, 2000.

[12] K. E. Aifantis and J. P. Dempsey, "Stable crack growth in nanostructured Li-batteries," Journal of Power Sources, vol. 143, no. 1-2, pp. 203-211, 2005.

[13] M. Ebner, F. Marone, M. Stampanoni, and V. Wood, "Visualization and quantification of electrochemical and mechanical degradation in Li ion batteries," Science, vol. 342, no. 6159, pp. 716-720, 2013.

[14] C. Lin, A. Tang, H. Mu, W. Wang, and C. Wang, "Aging mechanisms of electrode materials in lithium-ion batteries for electric vehicles," Journal of Chemistry, vol. 2015, Article ID 104673, 11 pages, 2015.

[15] S. J. Harris, R. D. Deshpande, Y. Qi, I. Dutta, and Y.-T. Cheng, "Mesopores inside electrode particles can change the Li-ion transport mechanism and diffusion-induced stress," Journal of Materials Research, vol. 25, no. 8, pp. 1433-1440, 2010.

[16] W. H. Woodford, Y.-M. Chiang, and W. C. Carter, "Electrochemical shock' of intercalation electrodes: a fracture mechanics analysis," Journal of the Electrochemical Society, vol. 157, no. 10, pp. A1052-A1059, 2010.

[17] S. K. Soni, B. W. Sheldon, X. Xiao, A. F. Bower, and M. W. Verbrugge, "Diffusion mediated lithiation stresses in Si Thin film electrodes," Journal of the Electrochemical Society, vol. 159, no. 9, pp. A1520-A1527, 2012.

[18] R. T. Purkayastha and R. M. McMeeking, "An integrated 2-D model of a lithium ion battery: the effect of material parameters and morphology on storage particle stress," Computational Mechanics, vol. 50, no. 2, pp. 209-227, 2012.

[19] J. Christensen, "Modeling diffusion-induced stress in Li-ion cells with porous electrodes," Journal of the Electrochemical Society, vol. 157, no. 3, pp. A366-A380, 2010.

[20] Y.-T. Cheng and M. W. Verbrugge, "Diffusion-induced stress, interfacial charge transfer, and criteria for avoiding crack initiation of electrode particles," Journal of the Electrochemical Society, vol. 157, no. 4, pp. A508-A516, 2010.

[21] Y.-T. Cheng and M. W. Verbrugge, "Application of Hasselman's crack propagation model to insertion electrodes," Electrochemical and Solid-State Letters, vol. 13, no. 9, pp. A128-A131, 2010.

[22] B. S. Haran, B. N. Popov, and R. E. White, "Determination of the hydrogen diffusion coefficient in metal hydrides by impedance spectroscopy," Journal of Power Sources, vol. 75, no. 1, pp. 56-63, 1998.

[23] E. Tatsukawa and K. Tamura, "Activity correction on electrochemical reaction and diffusion in lithium intercalation electrodes for discharge/charge simulation by single particle model," Electrochimica Acta, vol. 115, pp. 75-85, 2014.

[24] W. Fang, O. J. Kwon, and C.-Y. Wang, "Electrochemical-thermal modeling of automotive Li-ion batteries and experimental validation using a three-electrode cell," International Journal of Energy Research, vol. 34, no. 2, pp. 107-115, 2010.

[25] A. P. Schmidt, M. Bitzer, Á. W. Imre, and L. Guzzella, "Experiment-driven electrochemical modeling and systematic parameterization for a lithium-ion battery cell," Journal of Power Sources, vol. 195, no. 15, pp. 5071-5080, 2010.
[26] K. A. Smith, C. D. Rahn, and C.-Y. Wang, "Model-based electrochemical estimation and constraint management for pulse operation of lithium ion batteries," IEEE Transactions on Control Systems Technology, vol. 18, no. 3, pp. 654-663, 2010.

[27] Y. Qi, H. Guo, L. G. Hector Jr., and A. Timmons, “Threefold increase in the young's modulus of graphite negative electrode during lithium intercalation," Journal of the Electrochemical Society, vol. 157, no. 5, pp. A558-A566, 2010.

[28] S. P. Timoshenko and J. N. Goodier, Theory of Elasticity, McGraw-Hill, New York, NY, USA, 3rd edition, 1970.

[29] J. Crank, The Mathematics of Diffusion, Oxford, UK, Clarendon, 2nd edition, 1956.

[30] A. V. Shobukhov and D. S. Maximov, "Exact steady state solutions in symmetrical Nernst-Planck-Poisson electrodiffusive models," Journal of Mathematical Chemistry, vol. 52, no. 5, pp. 1338-1349, 2014 

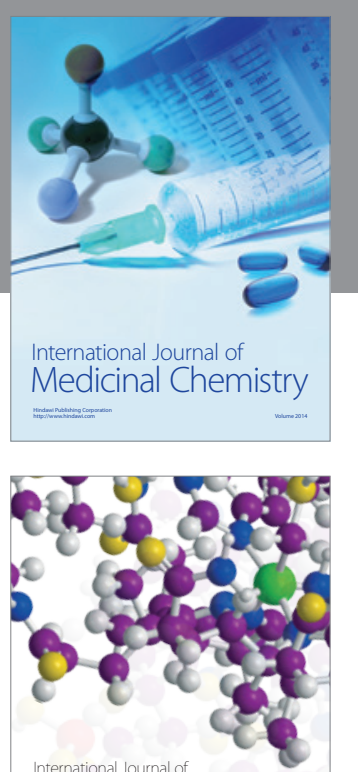

Carbohydrate Chemistry

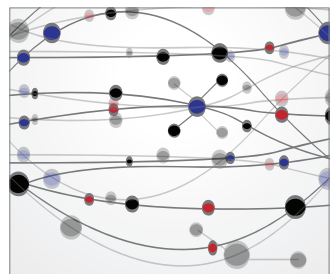

The Scientific World Journal
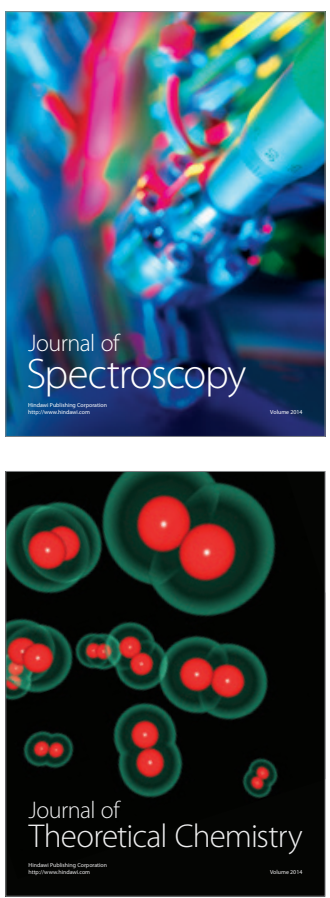
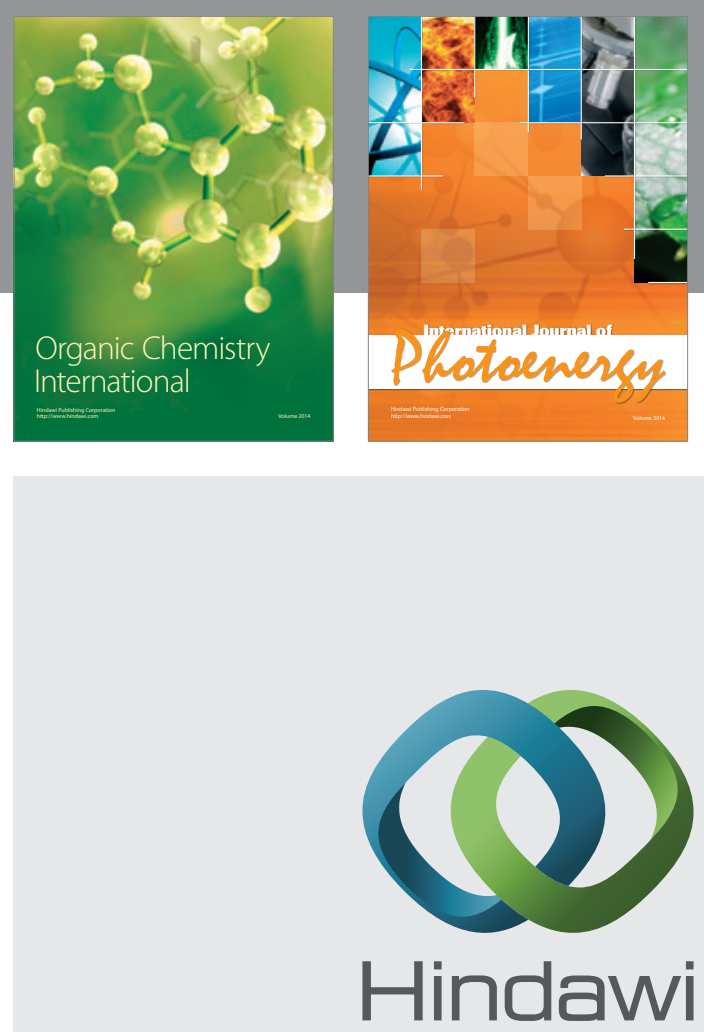

Submit your manuscripts at

http://www.hindawi.com

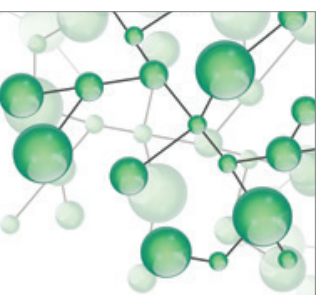

International Journal of

Inorganic Chemistry

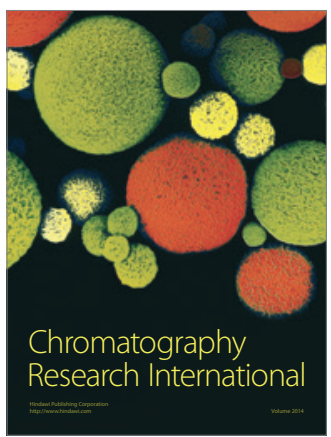

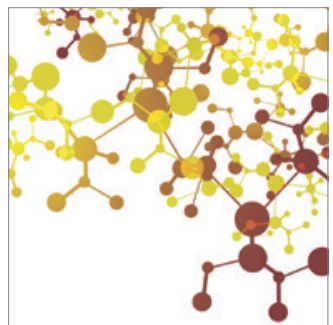

Applied Chemistry
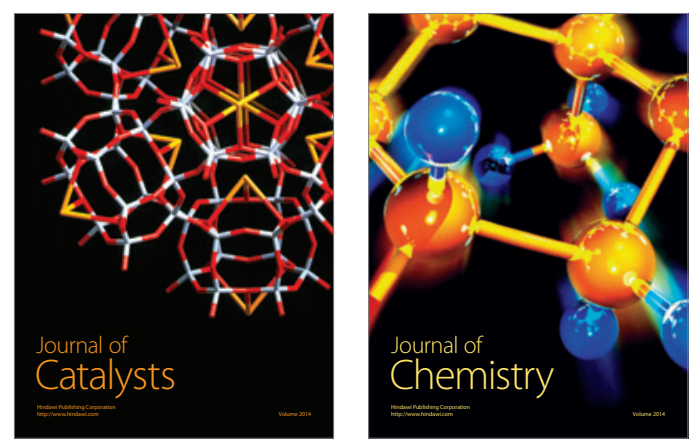
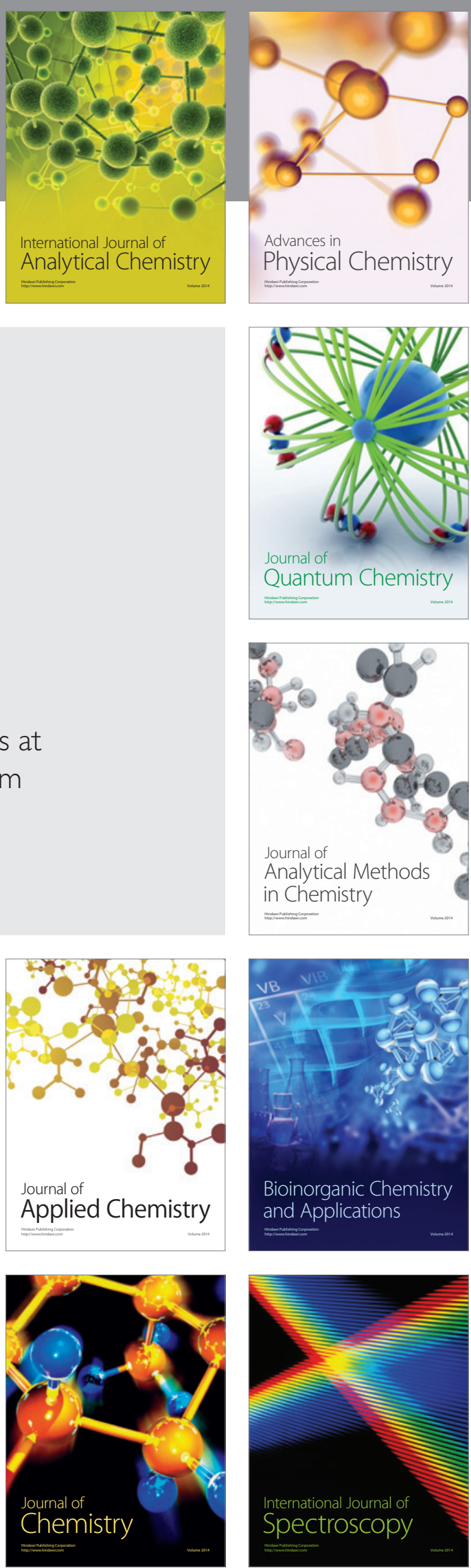